\title{
Clinical Comparison of Pencil Beam Convolution and Clarkson Algorithms for Dose Calculation
}

\author{
Abdulhamid Chaikh ${ }^{1,2}$, Jean-Yves Giraud ${ }^{1,2}$, Jacques Balosso ${ }^{1,2}$ \\ ${ }^{1}$ Joseph Fourier University, Grenoble, France; ${ }^{2}$ Department of Radiation Oncology and Medical Physics, Grenoble University Hos- \\ pital, Grenoble, France. \\ Email: abdulhamedc@yahoo.com \\ Received November $26^{\text {th }}, 2013$; revised December $17^{\text {th }}, 2013$; accepted December $23^{\text {rd }}, 2013$ \\ Copyright (C) 2013 Abdulhamid Chaikh et al. This is an open access article distributed under the Creative Commons Attribution Li- \\ cense, which permits unrestricted use, distribution, and reproduction in any medium, provided the original work is properly cited. In \\ accordance of the Creative Commons Attribution License all Copyrights (C) 2013 are reserved for SCIRP and the owner of the intel- \\ lectual property Abdulhamid Chaikh et al. All Copyright (C) 2013 are guarded by law and by SCIRP as a guardian.
}

\begin{abstract}
Purpose: The purpose of this work is to study and quantify the differences in calculated dose computed with two algorithms available in treatment planning systems: Pencil Beam Convolution and Clarkson. Material and Methods: Four different types of treatment cases were analyzed: lung, head and neck, brain and prostate. For each case, the volume definition was based on a clinical CT-scan acquisition. The patients were treated with 3-dimensional radiation therapy. For each patient, 2 treatment plans were generated using exactly the same configuration of beams. In plan 1 and plan 2 , the dose was calculated using the Clarkson and Pencil Beam Convolution algorithms, respectively, without heterogeneity correction. To evaluate the treatment plans, the monitor units, isodose curves, dose volume histograms and quality index were compared. A statistical analysis was carried out using Wilcoxon signed rank test. Results: The difference observed for monitor unites was $1.2 \%$ for lung and less than $1 \%$ for head and neck, brain and prostate. Wilcoxon test showed that there was "no statically significant difference, $(p>0.05)$ ". The dosimetric parameters derived from dose volume histograms were higher for organs at risks using Clarkson compared to Pencil Beam Convolution algorithm inviting clinician to make "safer" prescriptions. For quality index there was no statistically significant difference between both algorithms for all quality indexes, $(p>0.05)$. Conclusion: The clinical evaluation of a treatment plan should be made regarding the calculation algorithm which, in turn, is linked to the experience of the clinician.
\end{abstract}

Keywords: Treatment Planning; Dose Distribution; Isodose; Monitor Unit; PBC; Clarkson

\section{Introduction}

Treatment planning is one of the main steps in radiotherapy. It includes dose, isodose and monitor units (MUs) calculations. The dose calculation is based on algorithms implemented in treatment planning system (TPS). For a suitable clinical use, these algorithms must calculate the dose as accurately as possible. The radiotherapy department at Grenoble University Hospital was migrating from the Clarkson-Cunningham algorithm to the Pencil Beam Convolution (PBC) algorithm. Considering the change from one algorithm to the other, it was necessary to verify that the new algorithm will not introduce unexpected results in the clinical practice. So, we build a study to compare the two algorithms and quantify the differences in terms of dose to the patients. The comparisons between older and newer algorithm include four different clinical situations [1,2]. Monitor unit calculation is a very important step. MUs are directly related to the dose delivered to the patient. Specific task groups have recommended comparing the monitor unit calculation for different kinds of plans in order to confirm the validity of monitor unit calculations [3-5]. The aim of our study was to compare monitor units and dose distribution obtained with the two algorithms respectively: the Clarkson and the PBC algorithms, and to evaluate the possible change in the clinical outcome of treatment plans. The Clarkson method was the most widely spread method implemented in TPS. Most of the clinical trials conducted in radiation therapy over the last decade were done by center using this method. Furthermore, the values of dose limit for organ at risks OARs were certainly derived from these calculations. The PBC algorithm is now one of the most commonly available 
algorithms in commercial systems. In the present work, the dosimetric calculations of the two algorithms are investigated using four cancer cases. These cases were selected in our clinic. The treatment plans were designed with the treatment planning systems used in our clinic. The dose values calculated with the Clarkson algorithm are the references since this algorithm has been used for many years in our clinic.

\section{Material and Method}

\subsection{Planning CT Scans and Contouring of Structures}

In order to get the same $\mathrm{CT}$ images and the same contouring on each treatment planning systems for each clinical case, clinicians delineated the clinical contours of target structures and OARs on a specific workstation: $\mathrm{Imago}^{\circledR}$. We exported the CT images as well as the contours from Imago $^{\circledR}$ to respectively Dosigray ${ }^{\circledR}$ and Eclipse $^{\circledR}$ using a DICOM RT transfer. For each clinical case, we constructed similar treatment plans on each TPS. We checked the similarity of the treatment plans by comparing volume definitions, beam geometry and MLCsettings on each TPS. The treatment plans were designed using a forward planning method. The isocentre was the point where the dose was prescribed. The isodoses were normalized at the isocentre. It was located centrally into the tumor (center of the PTV). So, the dose is prescribed for each case at a single reference point inside the planning target volume as recommended by ICRU reports $[6,7]$.

\subsection{Treatment Planning Systems}

We used two treatment planning systems (TPS) in this study Dosigray ${ }^{\circledR}$ (Dosisoft) and Eclipse ${ }^{\circledR}$ (version 8.1.1.8, Varian medical systems). Dosigray ${ }^{\circledR}$ system uses a Clarkson model. The Clarkson method separates the dose into two components: primary and scattered radiations [8]. It resolves the irregular field into sectors of circular beams originating at the point of interest in the phantom or patient. The sector integration method calculates the dose as the sum of contributions of primary radiation and of scattered radiation. The Eclipse ${ }^{\circledR}$ system uses the PBC algorithm. This algorithm is based on a pencil beam kernel convolution. This algorithm compute the dose in the patient as the superposition of the total energy released per unit mass with an energy deposition kernel. Kernel represents the spread of energy from the primary photon interaction site throughout the volume [9].

\subsection{Prescription and Plans Settings for the Clinical Cases}

Lung: 12 field techniques with photon beam $18 \mathrm{MV}$ were used to treat a tumor placed in the mediastina. There were different gantry angles: 4 anteriors, 2 posteriors, 2 laterals, 4 obliques. The planning target volumes received a 60 Gy.

Head and neck: 12 field techniques with photon beam $6 \mathrm{MV}$ were used to treat a tumour placed in oral cavity. There were different gantry angles: six right and six left fields parallel and opposed two by two were used at $90^{\circ}$ and $270^{\circ}$ positions of the Linac arm. The planning target volumes received a $60.75 \mathrm{~Gy}$.

Brain: 5 oblique fields with photon beam $6 \mathrm{MV}$ were used to treat a right side tumor in the brain. The gantry angles were: $205^{\circ}, 345^{\circ}$ and $210^{\circ}$. The planning target volumes received a $36.4 \mathrm{~Gy}$.

Prostate: 10 field techniques with photon beam 18 MV were used to treat the seminal vesicles. Gantry directions were: 1 anterior, 1 posterior, 4 laterals, 4 obliques. The planning target volumes received a $70 \mathrm{~Gy}$.

\subsection{Treatment Plans Evaluation}

\subsubsection{Dosimetric Analysis}

In order to evaluate the treatment plans, the following dosimetric parameters were used:

1) MUs: for each patient, the MUs of plan 1 and 2 were compared for each field.

2) Isodose distribution: we compared the isodose curves $95 \%$ and $100 \%$ inside the PTVs.

3) Dose volume histograms (DVH): for each PTV the minimum dose, mean dose and maximum dose, as well as the calculated dose delivered to $95 \%$ of the PTV volume (D95) were compared. For each OAR, the minimum dose, mean dose and maximum dose were compared. We also compared the dose constraints for each organ at risks.

4) Quality index: Conformity Index (CI) defined as the ratio of the minimum dose encompassing the PTV to the prescribed dose was used to compare the plan conformity. Homogeneity Index (HI), defined as the ratio of the maximum dose in PTV to the prescribed dose was used to compare the homogeneity dose for PTV. The PTV Conformity Index (CIPTV), defined as the PTV volume receiving more than $95 \%$ of the prescribed dose divided by the PTV volume was used to compare the degree of conformity of the prescribed dose. We used the geometrical index ( $\mathrm{g}$ ) to compare the geometric conformity to PTV and normal tissues, where $\mathrm{g}=\left(\mathrm{V}_{\mathrm{PTV}}+\mathrm{V}_{\mathrm{NT}}\right) /$ PTV volumes. VPTV designates the PTV volumes receiving a dose lower than $100 \%$ the prescribed dose. VNT are the normal tissue volumes receiving $100 \%$ of the prescribed dose [9-11].

\subsubsection{Statistical Analysis}

Wilcoxon signed rank test was applied to assess the statically significance of deviations. Language $\mathrm{R}^{\circledR}$ (ver- 
sion 2.15.2/2012-10-26) was employed to calculate $p$ value at alpha error equal to $5 \%$. Data are presented as interquartile rang/Median; (IQR/M) or Mean \pm Standard Deviation (SD).

\section{Results}

\subsection{Planning CT Scans and Contouring of Structures}

We compared all structures obtained on each TPS and each treatment case. We did not found significant difference in the contours of volumes. Furthermore, the volumes of each structure, calculated by the two TPS from initial contouring are almost equal. This comparison demonstrated that, the same contours were used in both methods. So, our comparisons, even if conducted on two separate TPS, are based on the same volume definition.

\subsection{Comparison of Dosimetric Results}

1) MUs: the difference between the monitor units calculated by Clarkson and PBC algorithms were by a mean of $1.2 \%$ (1.8SD), $0.2 \%$ (0.9SD), $0.7 \%$ (1.6SD) and $0.2 \%$ (1.4SD) for lung, head and neck, brain and prostate, respectively. Wilcoxon test showed that there was no statically significant difference and $p$-values were 0.4 , 0.2, 0.8 and 0.7 for lung, head and neck, brain and prostate, respectively. Figure 1 shows the distribution of beam as a function of percentage of dose difference for all fields $(n=39)$. We note that using Clarkson algorithm in plan 1 compared to $\mathrm{PBC}$ algorithm in plan 2, the MUs were not changed for 23 fields, but the MUs were lower for 6 fields and higher for 10 fields.

2) Isodose distribution: the $95 \%$ isodose curves calculated in plan 1 and plan 2 included the whole PTVs whatever its location. The $100 \%$ isodose curves enclosed

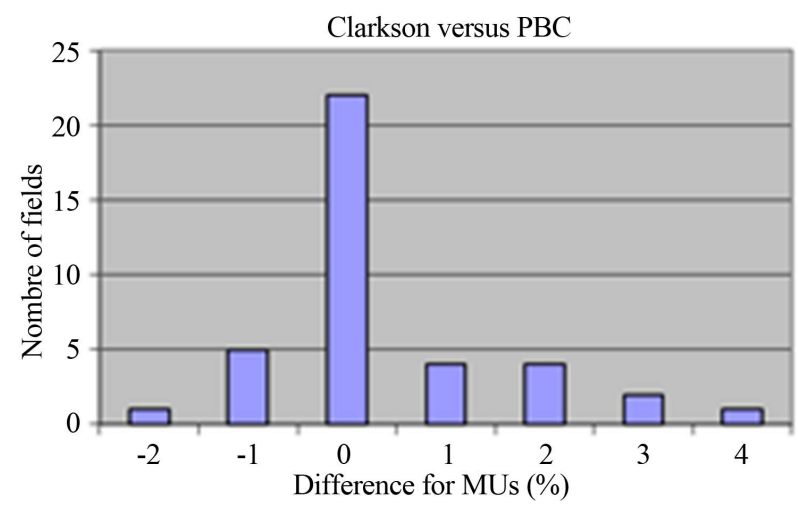

Figure 1. The distribution of beam as a function of percentage of dose difference per MUs for all fields. We note that using Clarkson algorithm in plans 1 compared to $\mathbf{P B C}$ algorithm in plans 2, the MUs were not changed for 23 fields, but the MUs were lower for 6 fields and higher for 10 fields. the PTVs using Clarkson more than PBC for brain and prostate. The $100 \%$ isodose curves enclosed the PTVs using PBC more than Clarkson for brain and prostate. Figure 2 shows the transverse view for lung, it can be seen that the $100 \%$ isodose line is larger on the PBC model than on the Clarkson calculation. On a clinical point of view, this could be evaluated as a better coverage of the inferior and lateral parts of the tumor volume than with the Clarkson algorithm. The Clarkson algorithm showed a reduced dose in the tumor. But with this algorithm the isodoses expand laterally more than with the PBC, showing to the clinician a higher dose to the OARs. We also note that the $20 \%$ line calculated by Clarkson method expands to the left lung (organ at risk) larger than PBC. This show more doses in the left lung than calculated by PBC. The $40 \%$ isodose line calculated by Clarkson algorithm expands longitudinally around the spinal cord. This shows a large dose near and within the spinal cord.

3) DVH: Tables 1 and $\mathbf{2}$ summarize the dosimetric and statistical results for PTVs and OARs. In Table 1, it

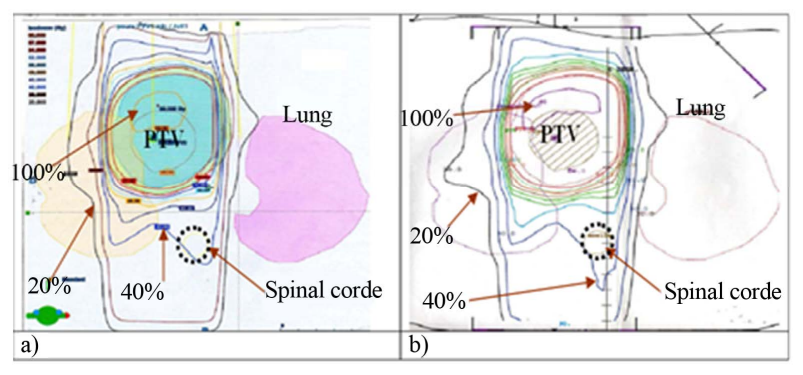

Figure 2. Transverse dose distribution for the lung cancer: a) PBC and b) Clarkson. The $20 \%$ and $40 \%$ isodose curves calculated by Clarkson in plan 1 encompass a greater volume of normal lung tissue and spinal cord, respectively, compared with PBC algorithm in plan 2.

Table 1. Dose volume parameters for planning target volume for all patients. $\Delta$ is the difference of values between plans 1 and plans 2. IQR/M: interquartile rang/Median; D95: the calculated dose delivered to $95 \%$ of the PTV volume and $p$-value: Wilcoxon signed rank test.

\begin{tabular}{ccccc}
\hline$\Delta$ Dose \% & Minimum dose & Mean dose & D95 & Maximum dose \\
\hline IQR/M & 1.2 & 0.6 & 4.4 & 0.4 \\
$\boldsymbol{p}$-value & $\mathbf{0 . 4}$ & $\mathbf{0 . 3}$ & $\mathbf{0 . 5}$ & $\mathbf{0 . 5}$ \\
\hline
\end{tabular}

Table 2. Dose volume parameters for all organs at risks for all patients. $\Delta$ is the difference of values between plans 1 and plans 2. IQR/M: interquartile rang/Median and $p$-value: Wilcoxon signed rank test.

\begin{tabular}{cccc}
\hline$\Delta$ Dose \% & Minimum dose & Mean dose & Maximum dose \\
\hline IQR/M & 1.6 & 4.0 & 2.0 \\
$\boldsymbol{p}$-value & $<\mathbf{0 . 0 0 1}$ & $\mathbf{0 . 0 2}$ & $<\mathbf{0 . 0 0 1}$ \\
\hline
\end{tabular}


can be seen that the difference between Clarkson and PBC algorithms for minimum, mean and maximum doses was less than $1 \%$. Wilcoxon test showed that there was no statically significant difference, $(p>0.05)$. In Table 2, it seems clearly that, the dose calculated for OARs by Clarkson algorithm was higher than PBC algorithm. The comparison of the dose constraints showed that the recommendation for dose constraints in all OARs were respected using the two algorithms.

4) Quality index: Table 3 summarizes the quality index for all patients using Clarkson and PBC algorithms. Wilcoxon test showed that there was no statistically significant difference for all index, $(p>0.05)$.

\section{Discussion}

Many papers evaluate the algorithms in terms of their abilities to accurately represent the dose distribution. Martel-Lafay et al. have recently recommended using an algorithm at least based on the superposition convolution method for calculating the dose in lung case [12]. Linthout et al demonstrated that Clarkson algorithm is suitable for the calculation of treatment plans for cranial and pelvic lesions [13]. For other anatomical regions like head and neck or lung, the Clarkson algorithm might no longer be sufficient. However, the reality of the representation of the dose distribution calculated by an algorithm has a clinical value when it is linked to the clinician experience. Our report presents a detailed analysis of the differences between the numbers of monitor unit, dose distribution, DVH and quality index for four treatment cases typologies calculated by two different algorithms.

While there are many similarities between the Clarkson algorithm and PBC algorithm in the calculation of monitors units, there are visible differences in dose values representation. The maximum doses calculated by the PBC algorithm were higher than with Clarkson for the lung and head and neck cases but lower for prostate and brain cases. The $100 \%$ and $95 \%$ isodoses levels calculated with the PBC method were better covering the target volume than those calculated with the Clarkson

Table 3. Quality index for all patients using Clarkson for plans 1 and PBC for plans 2. CI: Conformity Index; HI: Homogeneity Index; $\mathrm{CI}_{\mathrm{PTV}}$ : Conformity Index for planning target volume and g: geometrical index. p-value: Wilcoxon signed rank test. The results are shown here as Mean \pm Standard Deviation.

\begin{tabular}{ccccc}
\hline Index & $\mathrm{CI}$ & $\mathrm{HI}$ & $\mathrm{CI}_{\mathrm{PTV}}$ & $\mathrm{g}$ \\
\hline Clarkson & $0.8 \pm 0.3$ & $1 \pm 0$ & $0.9 \pm 0.2$ & $0.1 \pm 0.2$ \\
PBC & $0.8 \pm 0.3$ & $1.1 \pm 0.2$ & $0.8 \pm 0.2$ & $0.1 \pm 0.2$ \\
$\boldsymbol{p}$-value & $>\mathbf{0 . 0 5}$ & $>\mathbf{0 . 0 5}$ & $>\mathbf{0 . 0 5}$ & $>\mathbf{0 . 0 5}$ \\
\hline
\end{tabular}

method. However, the present study had the limitation that the population number of patients was small $(n=4)$. Whatever the differences are, clinician should not forget that these differences are only variations in the representtation of the dose distribution that exist in the patient. The only real distribution is the one that is clinically evaluated by the clinician.

\section{Conclusion}

This study enables physicians to be aware of treatment modifications associated with the change of dose calculation software using Clarkson and PBC algorithms without density correction. When changing from one algorithm to another, or when implementing recommenddations issued from other institutions or international trials, dose calculation methods must be carefully identified and evaluated prior to any clinical change in the prescription method.

\section{REFERENCES}

[1] Report of the Coordinated Research Project (CRP) on Development of Procedures for Quality Assurance of Dosimetry Calculations in Radiotherapy. Commissioning of Radiotherapy Treatment Planning Systems: Testing for Typical External Beam Treatment Techniques. IAEATECDOC-1583, 2008.

[2] A. M. Morgan, T. Knöös, S. G. McNee, C. J. Evans and D. I. Thwaites, "Clinical Implications of the Implementation of Advanced Treatment Planning Algorithms for Thoracic Treatments," Radiotherapy and Oncology, Vol. 86, No. 1, 2008, pp. 48-54.

http://dx.doi.org/10.1016/j.radonc.2007.11.033

[3] P. Lombardi, C. Fiorino, G. M. Cattaneo and R. Calandrino, "Monitor Unit Calculation in 6MV Irregularly Shaped Beam Accuracy in Clinical Practice," The British Journal of Radiology, Vol. 70, 1977, pp. 638-644.

[4] B. Fraass, K. Doppke, M. Hunt, G. Kutcher, G. Starkschall, R. Stern and J. Van Dyke, "AAPM Radiation Therapy Committee Task Group 53: Quality Assurance for Clinical Radiotherapy Treatment Planning," Medical Physics, Vol. 25, No. 10, 1998, pp. 1773-1829. http://dx.doi.org/10.1118/1.598373

[5] J. Chan, D. Russell, V. G. Peters and T. J. Farrell, "Comparison of Monitor Unit Calculations Performed with a 3D Computerized Planning System and Independent "Hand" Calculations: Results of Three Years Clinical Experience," Journal of Applied Clinical Medical Physics, Vol. 3, No. 4, 2002, pp. 293-301. http://dx.doi.org/10.1120/1.1506379

[6] International Commission on Radiation Units and Measurements, "ICRU Report No. 50: Prescribing, Recording and Reporting Photon Beam Therapy," Bethesda, 1993.

[7] International Commission on Radiation Units and Measurements, "ICRU Report No. 62: Prescribing, Recording and Reporting Photon Beam Therapy Supplement to ICRU Report 50," Bethesda, 1999. 
[8] E. B. Bengt, R. Harunor and H. O. Ceferino, "Separation of Primary and Scatter Components of Measured Photon Beam Data," Physics in Medicine and Biology, Vol. 34, No. 12, 1989, pp. 1939-1945.

http://dx.doi.org/10.1088/0031-9155/34/12/017

[9] A. Ahnesjö and M. M. Aspradakis, "Dose Calculations for External Photon Beams in Radiotherapy," Physics in Medicine and Biology, Vol. 44, No. 11, 1999, pp. 99-155. http://dx.doi.org/10.1088/0031-9155/44/11/201

[10] L. Feuvret, G. Noël, C. Nauraye, P. Garcia and J. J. Mazeron, "Conformal Index and Radiotherapy," Cancer/Radiothérapie, Vol. 8, No. 2, 2004, pp. 108-119. http://dx.doi.org/10.1016/j.canrad.2003.12.002

[11] N. J. Lomax and S. G. Scheib, "Quantifying the Degree of Conformity in Radiosurgery Treatment Planning," International Journal of Radiation Oncology, Biology, Physics, Vol. 55, No. 5, 2003, pp. 1409-1419. http://dx.doi.org/10.1016/S0360-3016(02)04599-6

[12] I. Martel-Lafay "Best Practice Guide for Radiation Therapy of Non-Small Cell Bronchial Cancers," Cancer/Radiothérapie, Vol. 13, No. 1, 2009, pp. 55-60.

[13] L. Nadine, V. Dirk, V. A. Swana, V. Mia, B. Anette and S. Guy, "Evaluation of Dose Calculation Algorithms for Dynamic Arc Treatments of Head and Neck Tumors," Radiotherapy and Oncology, Vol. 64, No. 1, 2002, pp. 85-95. http://dx.doi.org/10.1016/S0167-8140(02)00146-9 